\title{
Iterative Transmitter and Receiver Optimization for CDMA Networks
}

\author{
Sennur Ulukus, Member, IEEE and Aylin Yener, Member, IEEE
}

\begin{abstract}
Optimization of the capacity of a single-cell code-division multiple-access (CDMA) system, both from the perspective of the maximum number of users that can be served at a required quality of service level and from the information theoretic perspective, has been recently shown to be achieved by the same joint transmit and receive strategies. In this paper, we propose an alternating minimization based iterative algorithm that updates the transmitters and the corresponding receivers of the users. The algorithm is suitable for online implementation, and the objective function is suitable for extension to multicell networks, both of which are in contrast with the previously proposed algorithms. We show that the algorithm is provably convergent to the optimum signature sequences and the corresponding receivers.
\end{abstract}

Index Terms-Alternating minimization, code-division multiple access (CDMA), mean squared error (MSE), optimum signature sequence sets, sum capacity, Welch bound equality (WBE) sequences.

\section{INTRODUCTION}

C APACITY of code-division multiple-access (CDMA) systems has been studied extensively [3]-[5]. Reference [3] defines the user capacity of a CDMA cell as the number of users that can be accommodated at the required quality of service level defined in terms of the signal-to-interference ratio (SIR), and computes the user capacity when random signature sequences are used in a large system, for matched filter receivers and for minimum mean squared error (MMSE) receivers [6]. The information theoretic capacity region of a synchronous CDMA cell was derived in [4] for a given deterministic set of signature sequences. Reference [7] identified the set that maximizes the information theoretic sum capacity with equal power users, as one where all signature sequences are orthogonal to each other if possible, i.e., when the number of users is less than or equal to the processing gain, and as the Welch bound equality (WBE) sequences, otherwise. More recently [8] identified the optimum signature sequences for arbitrary (unequal) powers. An important result that bridges the two capacity results is given by [5] where user capacity is considered for deterministic and finite dimensional signature sequence sets. It is shown that, for a single CDMA cell where users have the same required SIR level, the capacity is achieved with minimum total power by each user having equal received powers and using the signature sequence sets that are identified in [7]. Furthermore, when such sets are

Manuscript received March 20, 2002; revised January 13, 2003. The editor coordinating the review of this paper and approving it for publication is S. Roy. This work was supported by the National Science Foundation under Grants ANI 02-05330 and CCR 02-37727. This work was presented in part at CISS 2002 [1] and IEEE ISIT 2002 [2].

S. Ulukus is with the Department of Electrical and Computer Engineering, University of Maryland, College Park, MD 20742 USA (e-mail: ulukus@eng.umd.edu).

A. Yener is with the Electrical Engineering Department, Pennsylvania State University, University Park, PA16802 USA (e-mail: yener@ee.psu.edu).

Digital Object Identifier 10.1109/TWC.2004.837453 used, the MMSE filters reduce to scalar multiples of matched filters [5].

Following these developments, iterative algorithms that converge to the optimum signature sequence sets are proposed [9]-[11]. Both algorithms proposed in [9]-[11], the MMSE algorithm [9], [10], and the eigen algorithm [11] work on minimizing the total (weighted) squared correlation, T(W)SC. The algorithms are distributed and their convergence can be proven only when the algorithms are run off-line and in a sequential manner. In this paper, we develop iterative transmitter and receiver design algorithms, based on the optimization of the MSE function. The major difference of the MSE from the sum capacity and the TWSC is that the MSE depends on both the transmitters (signature sequences) and the receivers (receiver filters). As we will see, even though minimizing the MSE, minimizing the TWSC, and maximizing the sum capacity all yield the same transmitter-receiver pairs in single-cell synchronous CDMA systems, minimizing the MSE produces an alternative iterative algorithm that offers two important advantages over the existing algorithms that minimize the TWSC: it enables online and parallel implementations.

The TWSC is a function of the signature sequences only. Algorithms based on the minimization of the TWSC [9]-[11] assume either of the following.

a) As the signature sequences are updated, the receiver filters are changed to be the corresponding matched filters instantaneously.

b) The iterations are run off-line only in terms of the signature sequences, and once the signature sequences converge to an optimum set, corresponding matched filters are deployed as receivers.

Therefore, the existing transmitter design algorithms are not amenable to an online implementation. In addition, the convergence proofs of the existing algorithms [9]-[11] require that only one user updates its signature sequence at a time. Therefore, such algorithms require sequential updates, which necessitate a careful scheduling of the user updates. Hence, the existing transmitter design algorithms are not amenable to parallel implementations. The introduction of the MSE alleviates both of these problems. By enabling online implementation, the adaptive [6], [12], [13] and blind-adaptive [14] implementations of the MMSE receiver may be used, and by enabling parallel updates, users would have the flexibility of updating their signature sequences in a sequential or a parallel fashion. Even though our model in this paper is strictly limited to a single-receiver (single-cell) system, another advantage of the MSE cost function is that it can be generalized to the multireceiver (multicell) networks more readily then the TWSC, since the MSE cost function can account for the different channel gains of the users to multiple receiver sites. 
In the following, we develop an iterative transmitter-receiver update algorithm that is based on the alternating minimization technique on the MSE cost function, and we prove its convergence to optimum transmit-receiver pairs with online and parallel user updates.

\section{System Model And Performance Metric}

We consider the uplink of a single cell synchronous CDMA system with $K$ users and processing gain $N$. In the presence of additive white Gaussian noise, the received vector signal in one symbol interval is

$$
\mathbf{r}=\sum_{i=1}^{K} \sqrt{p_{i}} b_{i} \mathbf{s}_{i}+\mathbf{n}
$$

where, for user $i, p_{i}$ is the received power, $b_{i}$ is the information symbol, and $\mathbf{s}_{i}$ is the unit-energy signature sequence, and $\mathbf{n}$ is a zero-mean Gaussian random vector with $E\left[\mathbf{n n}^{\top}\right]=\sigma^{2} \mathbf{I}_{N}$, where $\mathbf{I}_{N}$ denotes the $N \times N$ identity matrix.

Previous work showed that the information theoretic sum capacity of this system is given by [4]

$$
C_{\text {sum }}=\frac{1}{2} \log \left[\operatorname{det}\left(\mathbf{I}_{N}+\sigma^{-2} \mathbf{S P S}^{\top}\right)\right]
$$

where $\mathbf{S}=\left[\mathbf{s}_{1}, \ldots, \mathbf{s}_{K}\right]$ is an $N \times K$ matrix with the users' signature sequences as its columns and $\mathbf{P}=\operatorname{diag}\left\{p_{1}, \ldots, p_{K}\right\}$ is a $K \times K$ diagonal matrix of the users' received powers. When the received powers of the users are the same, $p_{i}=p$ for all $i$, $C_{\text {sum }}$ is maximized by [7]

$$
\mathbf{S}^{\top} \mathbf{S}=\mathbf{I}_{K}
$$

if $K \leq N$ and by

$$
\mathbf{S S}^{\top}=\frac{K}{N} \mathbf{I}_{N}
$$

if $K>N$. The signature sequence sets satisfying (3) contain $K$ orthonormal signature sequences in $N$-dimensional vector space, and the sequence sets satisfying (4) are the WBE sequences [7].

For arbitrary (unequal) powers, the signature sequences that maximize the sum capacity in (2) are identified in [8]. It was shown that, when $K \leq N, C_{\text {sum }}$ is again maximized by orthonormal sequences. In the case of $K>N$, the capacity is maximized when users with relatively high received powers, termed as oversized users in [8], are assigned sequences orthogonal to all other users, and the remaining users are assigned generalized WBE sequences in the reduced dimensionality signal space [8]. Assuming that the users are ordered according to their powers $p_{1}>\cdots>p_{K}$ and the first $L$ users are oversized, the eigenvalues of $\mathbf{S P S}^{\top}$ with the optimum $\mathbf{S}$ are $\left\{p_{1}, \ldots, p_{L}, \lambda, \ldots, \lambda\right\}$, where $\lambda=\left(\sum_{l=L+1}^{K} p_{l}\right) /(N-L)$, and the multiplicity of $\lambda$ is $N-L$. In other words, the characterization of the optimum signature sequences is that the eigenvalues of $\mathbf{S P S}^{\top}$ with the optimum signature sequences are majorized by the eigenvalues of the same matrix with any other feasible signature sequence set. The eigenvalues of
$\mathbf{S P S}^{\top}$ corresponding to the optimum signature sequences is a Schur-minimal element of the space of all feasible eigenvalues corresponding to all possible signature sequence sets. We will use the fact that a Schur-minimal point maximizes all Schur-concave functions and minimizes all Schur-convex functions in the sequel [15].

The MSE incurred by a user, say, user $i$, at the output of a linear filter $\mathbf{c}_{i}$ is

$$
\begin{aligned}
\operatorname{MSE}_{i} & =E\left[\left(\mathbf{r}^{\top} \mathbf{c}_{i}-b_{i}\right)^{2}\right] \\
& =\mathbf{c}_{i}^{\top}\left(\mathbf{S P S}^{\top}+\sigma^{2} \mathbf{I}_{N}\right) \mathbf{c}_{i}-2 \sqrt{p_{i}} \mathbf{c}_{i}^{\top} \mathbf{s}_{i}+1 .
\end{aligned}
$$

Let us define the total mean squared error of the system as

$$
\begin{aligned}
\text { MSE } & =\sum_{i=1}^{K} \operatorname{MSE}_{i} \\
& =\operatorname{tr}\left[\mathbf{C}^{\top}\left(\mathbf{S P S} \mathbf{S}^{\top}+\sigma^{2} \mathbf{I}_{N}\right) \mathbf{C}-2 \mathbf{C}^{\top} \mathbf{S} \mathbf{P}^{1 / 2}+\mathbf{I}_{K}\right](6)
\end{aligned}
$$

where $\mathbf{C}=\left[\mathbf{c}_{1}, \ldots, \mathbf{c}_{K}\right]$ is an $N \times K$ matrix containing the receiver filters of the users in its columns. Consider the MMSE filters for all users for fixed signature sequences. In this case $\mathbf{C}$ is given as

$$
\mathbf{C}=\left(\mathbf{S P S}^{\top}+\sigma^{2} \mathbf{I}_{N}\right)^{-1} \mathbf{S P}^{1 / 2} .
$$

Substituting (7) into (6), we obtain the total MMSE of the system with signature sequence set $\mathbf{S}$ as

$$
\text { MMSE }=K-\operatorname{tr}\left[\mathbf{S P S}^{\top}\left(\mathbf{S P S}^{\top}+\sigma^{2} \mathbf{I}_{N}\right)^{-1}\right] .
$$

The TWSC corresponding to this system is

$$
\operatorname{TWSC}=\sum_{i=1}^{K} \sum_{j=1}^{K} p_{i} p_{j}\left(\mathbf{s}_{i}^{\top} \mathbf{s}_{j}\right)^{2}=\operatorname{tr}\left[\left(\mathbf{S P S}^{\top}\right)^{2}\right] .
$$

In terms of the eigenvalues of $\mathbf{S P S}^{\top},\left\{\lambda_{i}\right\}$

$$
\begin{aligned}
C_{\text {sum }} & =\frac{1}{2} \sum_{i=1}^{N} \log \left(1+\frac{\lambda_{i}}{\sigma^{2}}\right) \\
\text { MMSE } & =K-\sum_{i=1}^{N} \frac{\lambda_{i}}{\lambda_{i}+\sigma^{2}} \\
\text { TWSC } & =\sum_{i=1}^{N} \lambda_{i}^{2} .
\end{aligned}
$$

It is easy to see that $C_{\text {sum }}$ is Schur-concave and MMSE and TWSC are Schur-convex functions of the eigenvalues of SPS $^{\top}$ [15]. Therefore, the signature sequence matrix yielding Schur-minimal eigenvalues, i.e., one with eigenvalues that are majorized by all other feasible eigenvalues, which maximizes $C_{\text {sum }}$, also minimizes the MMSE and the TWSC.

Since our aim is to obtain an online iterative joint receiver-transmitter update algorithm, we will concentrate on the MSE criterion given in (6), which is a function of all signature sequences and receiver filters. Clearly, the minimization of (6) 
over the signature sequences and the receiver filters is equivalent to the minimization of the MMSE in (8) over the signature sequences. This, in turn, is also equivalent to the minimization of TWSC and the maximization of $C_{\text {sum }}$ over the signature sequences.

\section{An Alternating Minimization Algorithm AND ITS CONVERGENCE}

Our aim is to minimize the cost function in (6) over the signature sequences $\left\{\mathbf{s}_{i}\right\}$ and the receivers $\left\{\mathbf{c}_{i}\right\}$. We assume the received powers are given, and require the resulting signature sequences to have unit energy. That is, we impose the set of constraints $\mathbf{s}_{i}^{\top} \mathbf{s}_{i}=1$ for all $i=1, \ldots, K$. The Lagrangian for this constrained optimization problem is expressed as

$$
\begin{aligned}
\mathcal{L}\left(\left\{\mathbf{c}_{i}\right\},\left\{\mathbf{s}_{i}\right\},\left\{\mu_{i}\right\}\right)= & \sum_{i=1}^{K} \sum_{j=1}^{K} p_{j}\left(\mathbf{c}_{i}^{\top} \mathbf{s}_{j}\right)^{2}-2 \sum_{i=1}^{K} \sqrt{p_{i}}\left(\mathbf{c}_{i}^{\top} \mathbf{s}_{i}\right) \\
& +\sigma^{2} \sum_{i=1}^{K} \mathbf{c}_{i}^{\top} \mathbf{c}_{i}+\sum_{i=1}^{K} \mu_{i}\left(\mathbf{s}_{i}^{\top} \mathbf{s}_{i}-1\right)
\end{aligned}
$$

where $\left\{\mu_{i}\right\}$ are the Lagrange multipliers. One can devise an iterative algorithm to optimize this function based on the block coordinate descent method, also known as alternating minimization [16]. The idea is to fix the value of all but one of the vector variables in the function and optimize over that variable. One then iterates between different variables optimizing one at a time.

Consider the receiver filters first. As mentioned before, minimization of the total MSE with respect to the receiver filter of user $i$ is equivalent to minimizing $\mathrm{MSE}_{i}$. This is a simple consequence of the fact that a user's receiver does not affect the MSE of any other user but itself. Thus, if we keep the signature sequences fixed, all receivers need to be set to the MMSE filters for all users

$$
\mathbf{c}_{i}=\sqrt{p_{i}}\left(\mathbf{S P S}^{\top}+\sigma^{2} \mathbf{I}_{N}\right)^{-1} \mathbf{s}_{i}
$$

Note that all users can update their receivers in a parallel fashion, since the receiver updates are independent of each other.

Next, consider the signature sequence updates. Once again, for user $i$, we need to optimize the Lagrangian by keeping all other variables fixed. The signature sequence update for user $i$ is found as

$$
\mathbf{s}_{i}=\sqrt{p_{i}}\left(p_{i} \mathbf{C} \mathbf{C}^{\top}+\mu_{i} \mathbf{I}_{N}\right)^{-1} \mathbf{c}_{i}
$$

where $\mu_{i}$ is the Lagrange multiplier chosen such that the resulting signature sequence $\mathbf{s}_{i}$ is of unit energy. Note that the signature sequence update of user $i$ depends on all users' receiver filters and not the signature sequences of the other users. Thus, all users can update their signature sequences in parallel as well. Note also that each signature sequence update closely resembles an MMSE-type update. More specifically, the update replaces the signature sequence of the $i$ th user by a generalized MMSE receiver filter of a system where the signature sequences of the users are their receiver filters, and all users have equal powers equal to the power of user $i, p_{i}$. Lastly, we note that the signature sequence update is similar to one of the updates proposed in [17] for multipath channels. Iterative algorithms that aim at minimizing the total MSE have previously been presented in [17] and [18] without any claims on their convergence.

Our algorithm starts with arbitrary initial signature sequences $(\mathbf{S})$ and receiver filters $(\mathbf{C})$, updates $\mathbf{S}$ for fixed $\mathbf{C}$, updates $\mathbf{C}$ for fixed (updated) $\mathbf{S}$, etc. The convergence of the overall algorithm is established by first observing that each update decreases the total MSE function which is bounded from below, and then investigating the properties of the fixed points of the algorithm. Let us consider a complete cycle of signature sequence and receiver filter updates for all users $\mathbf{S} \rightarrow \mathbf{C} \rightarrow \hat{\mathbf{S}}$. Using (12) and (13)

$$
\hat{\mathbf{s}}_{i}=\left[\mathbf{S P S}^{\top}\left(\mathbf{S P S}^{\top}+\sigma^{2} \mathbf{I}_{N}\right)^{-1}+\frac{\mu_{i}}{p_{i}}\left(\mathbf{S P S}^{\top}+\sigma^{2} \mathbf{I}_{N}\right)\right]^{-1} \mathbf{s}_{i}
$$

At the fixed point of the algorithm $\hat{\mathbf{s}}_{i}=\mathbf{s}_{i}$, for all $i$. This implies that the signature sequence of user $i, \mathbf{s}_{i}$, should be an eigenvector of $\mathbf{B}_{i}$ with eigenvalue 1 . Moreover, we note that the eigenvectors of $\mathbf{B}_{i}$ are the eigenvectors of $\mathbf{S P S}^{\top}$. Therefore, the fixed-point signature sequences satisfy

$$
\mathbf{S P S}^{\top} \mathbf{s}_{i}=\lambda_{i} \mathbf{S}_{i} \quad i=1, \ldots, K .
$$

When the signature sequences satisfy (15), the corresponding fixed-point receiver filters obtained from $\mathbf{S}$ using (12) are scaled matched filters, i.e., $\mathbf{c}_{i}=k_{i} \mathbf{s}_{i}$.

Equation (15) gives a complete description of the set of all possible fixed points. Fixed points are those for which MSE or SIR cannot be improved by linear filtering for any user, i.e., MMSE filters are scaled matched filters. Unfortunately, the set of fixed points described by (15) includes a wide spectrum of signature sequences, ranging from the very best signature sequences to which we want our algorithm to converge, to the absolutely worst signature sequences. For instance, the all-equal signature sequences, i.e., $\mathbf{s}_{i}=\mathbf{s}$, which may be considered to be the worst signature sequences as a set, satisfy (15). Note also that the best signature sequences that maximize $C_{\text {sum }}$ and minimize MSE and TWSC satisfy (15) too [10], [11], [19].

Next, we note that the fixed points of our algorithm are the same as the fixed points of the MMSE algorithm proposed in [10] which minimizes the TWSC. It was noted in [10] that when the algorithm was started from a set of randomly generated initial signature sequences, it always converged to an optimum signature sequence set. This claim is supported by a complete convergence proof in a recent paper [19]. Reference [19] proves that the suboptimum fixed points of the MMSE algorithm of [10] are unstable, in that, if the fixed point is not a global optimum solution, then one can find a signature sequence set in the $\epsilon$-neighborhood of the fixed-point signature sequence set which has strictly lower TWSC. Consequently, the almost sure convergence of the MMSE algorithm to a globally optimum signature sequence set is ensured by defining a noisy version of the algorithm in [10], where a small noise is added 
to the deterministic updates. In a similar approach, [20] gives a method of ensuring the convergence of the eigen algorithm of [11] to a global optimum point. Reference [20] notes that the TWSC at the fixed points of the eigen algorithm may take only a finite number of possible values as each suboptimal fixed point corresponds to an orthogonal partitioning of the signature sequence set. Then, [20] provides escape mechanisms from the suboptimum fixed points; since there are only a finite number of TWSC levels, the convergence to a global optimum is guaranteed. The escape mechanisms of [20] and $\epsilon$-perturbation ideas of [19] are similar; both are based on the idea of connecting the orthogonal partitions, i.e., perturbing the signature sequences in the orthogonal sets in a way to destroy the orthogonality between the sets.

We will use the same ideas here in order to show that the suboptimum fixed points of the proposed algorithm are unstable; hence, a noisy version of the proposed algorithm would ensure the almost sure convergence to a globally optimum signature sequence set and the corresponding receivers. We will follow [19] and provide the outline of how the results of [19] may be modified to complete the convergence proof here. To that end, we modify the results proved for the TWSC (called $\mathrm{TSC}_{g}$ in [19]) in [19, Lemmas 14 and 16] for our cost function MSE. The result in [19, Lemma 15], which is also necessary for the global convergence in general, is not needed here, as we assumed that the Gaussian noise is white, i.e., the eigenvalues of the noise covariance matrix are all equal. However, we note that our algorithm and results can be generalized straightforwardly to the colored noise case, and in that case, [19, Lemma 15] can be easily proved for the MSE cost function.

Assume that the signature sequences are partitioned into orthogonal sets. Let user 1 with signature sequence $\mathbf{s}_{1}$, power $p_{1}$, and eigenvalue $\lambda_{1}$ be in set $\mathbf{S}_{1}$, and user 2 with signature sequence $\mathbf{S}_{2}$, power $p_{2}$, and eigenvalue $\lambda_{2}$ be in set $\mathbf{S}_{2}$. All of the signature sequences in set $\mathbf{S}_{1}$ are orthogonal to all of the signature sequences in $\mathbf{S}_{2}$, and within each set, all users have the same eigenvalue. As in [19, Lemma 14], if $\lambda_{1}>\lambda_{2}$, then we should have $p_{1} \geq p_{2}$; otherwise we can perturb the signature sequences $\mathbf{s}_{1}$ and $\mathbf{s}_{2}$ slightly to decrease the MSE cost function. Assume otherwise, i.e., $\lambda_{1}>\lambda_{2}$ and $p_{1}<p_{2}$, and use the same perturbation as in [19, Lemma 14]

$\overline{\mathbf{s}}_{1}=\sqrt{1-\alpha^{2}} \mathbf{s}_{1}+\alpha \mathbf{s}_{2} \quad$ and $\quad \overline{\mathbf{s}}_{2}=\sqrt{1-\beta^{2}} \mathbf{s}_{2}+\beta \mathbf{s}_{1}$

where $\beta=-\left(p_{1} / p_{2}\right) \alpha$, and $\alpha=\sin \epsilon$. Note that $\overline{\mathbf{s}}_{1}^{\top} \overline{\mathbf{s}}_{1}=1$, $\overline{\mathbf{s}}_{2}^{\top} \overline{\mathbf{s}}_{2}=1$, and

$$
\overline{\mathbf{S}} \mathbf{P} \overline{\mathbf{S}}^{\top}=\mathbf{S P S}^{\top}+\Delta
$$

where

$$
\begin{aligned}
\boldsymbol{\Delta}= & \left(\beta^{2} p_{2}-\alpha^{2} p_{1}\right)\left(\mathbf{s}_{1} \mathbf{s}_{1}^{\top}-\mathbf{s}_{2} \mathbf{s}_{2}^{\top}\right) \\
& +\left(p_{1} \alpha \sqrt{1-\alpha^{2}}+p_{2} \beta \sqrt{1-\beta^{2}}\right)\left(\mathbf{s}_{1} \mathbf{s}_{2}^{\top}+\mathbf{s}_{2} \mathbf{s}_{1}^{\top}\right) .
\end{aligned}
$$

Reference [19, Lemma 14] shows that, with this perturbation

$\operatorname{TWSC}(\mathbf{S})-\operatorname{TWSC}(\overline{\mathbf{S}})=2 \frac{p_{1}}{p_{2}}\left(\lambda_{1}-\lambda_{2}\right)\left(p_{2}-p_{1}\right) \epsilon^{2}+O\left(\epsilon^{3}\right)$ and, therefore, that the TWSC can be reduced if $p_{1}<p_{2}$ while $\lambda_{1}>\lambda_{2}$. We assume that, after we perturb the signature sequences in the same way, i.e., as in (16), we let all users update their receiver filters (from $\mathbf{C}$, which were scaled matched filters for the signature sequences $\mathbf{S}$, to some $\overline{\mathbf{C}}$, which are the MMSE receivers for the perturbed signature sequences). It can be shown by straightforward calculations that

$$
\begin{aligned}
& \operatorname{MSE}(\mathbf{S}, \mathbf{C})-\operatorname{MSE}(\overline{\mathbf{S}}, \overline{\mathbf{C}}) \\
& \quad=\operatorname{MMSE}(\mathbf{S})-\operatorname{MMSE}(\overline{\mathbf{S}}) \\
& \quad=\sigma^{2} \frac{p_{1}}{p_{2}}\left(\frac{1}{\left(\lambda_{2}+\sigma^{2}\right)^{2}}-\frac{1}{\left(\lambda_{1}+\sigma^{2}\right)^{2}}\right)\left(p_{2}-p_{1}\right) \epsilon^{2}+O\left(\epsilon^{3}\right)
\end{aligned}
$$

and, therefore, that the MSE can be reduced if $p_{1}<p_{2}$ while $\lambda_{1}>\lambda_{2}$.

Assume again that the signature sequences are partitioned into orthogonal sets. Let users in $\mathbf{S}_{1}$ have the eigenvalue $\lambda_{1}$, and let us define $\mathbf{P}_{1}$ as the diagonal matrix that contains the powers of users in $\mathbf{S}_{1}$ on its diagonal. As in [19, Lemma 16], if $\lambda_{1}>\lambda_{2}$, where $\lambda_{2}$ denotes the eigenvalues of users in another orthogonal set $\mathbf{S}_{2}$, then the number of users in set $\mathbf{S}_{1}$ should be smaller than or equal to the rank of set $\mathbf{S}_{1}$; otherwise, we can perturb all of the signature sequences in set $\mathbf{S}_{1}$ slightly to decrease the MSE cost function. Assume otherwise, i.e., $\lambda_{1}>\lambda_{2}$, and the number of users in $\mathbf{S}_{1}$ is larger than the rank of the set, and use the same perturbation as in [19, Lemma 16]

$$
\overline{\mathbf{s}}_{k}=\cos \left(\alpha_{k}\right) \mathbf{s}_{k}+\sin \left(\alpha_{k}\right) \mathbf{q}
$$

for $\mathbf{s}_{k} \in \mathbf{S}_{1}$ and $\overline{\mathbf{s}}_{k}=\mathbf{s}_{k}$, otherwise. Here, $\mathbf{q}$ is an eigenvector of $\mathbf{S P S}^{\top}$ that is orthogonal to the signature sequences of users in $\mathbf{S}_{1}$ and belongs to the dimensions of set $\mathbf{S}_{2}$. We choose $\alpha_{k}=$ $\epsilon v_{k}$, where $\left\{v_{k}\right\}$ are the components of a vector that is chosen to satisfy $\mathbf{S}_{1} \mathbf{P}_{1} \mathbf{v}=\mathbf{0}$. Such a $\mathbf{v}$ exists because the signature sequences in $\mathbf{S}_{1}$ are assumed to be linearly dependent.

Reference [19, Lemma 16] shows that, with this perturbation

$$
\operatorname{TWSC}(\mathbf{S})-\operatorname{TWSC}(\overline{\mathbf{S}})=2\left(\lambda_{1}-\lambda_{2}\right)\left\|\mathbf{P}_{1} \mathbf{v}\right\|^{2} \epsilon^{2}+O\left(\epsilon^{3}\right)
$$

and therefore, that the TWSC can be reduced if $\lambda_{1}>\lambda_{2}$. We assume, as in the generalization of [19, Lemma 14] to the MSE cost function, that after we perturb the signature sequences, we let all users update their receiver filters from $\mathbf{C}$ to $\overline{\mathbf{C}}$, the MMSE filters corresponding to $\overline{\mathbf{S}}$. Then, it can be shown by straightforward calculations that

$$
\begin{aligned}
& \operatorname{MSE}(\mathbf{S}, \mathbf{C})-\operatorname{MSE}(\overline{\mathbf{S}}, \overline{\mathbf{C}}) \\
& \quad=\operatorname{MMSE}(\mathbf{S})-\operatorname{MMSE}(\overline{\mathbf{S}}) \\
& \quad=\sigma^{2}\left(\frac{1}{\left(\lambda_{2}+\sigma^{2}\right)^{2}}-\frac{1}{\left(\lambda_{1}+\sigma^{2}\right)^{2}}\right)\left\|\mathbf{P}_{1} \mathbf{v}\right\|^{2} \epsilon^{2}+O\left(\epsilon^{3}\right)
\end{aligned}
$$

and, therefore, that, the MSE can be reduced if $\lambda_{1}>\lambda_{2}$.

These results can be combined as in [19, Theorems 6 and 7] to conclude that the MSE cost function does not have local minima; that is, there is always a signature sequence set and 


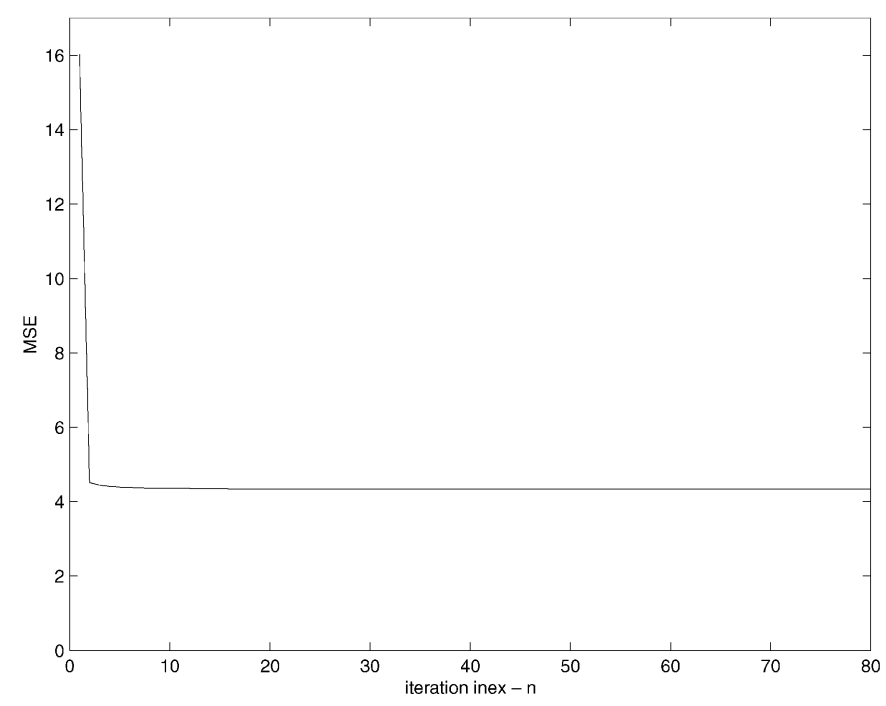

(a)

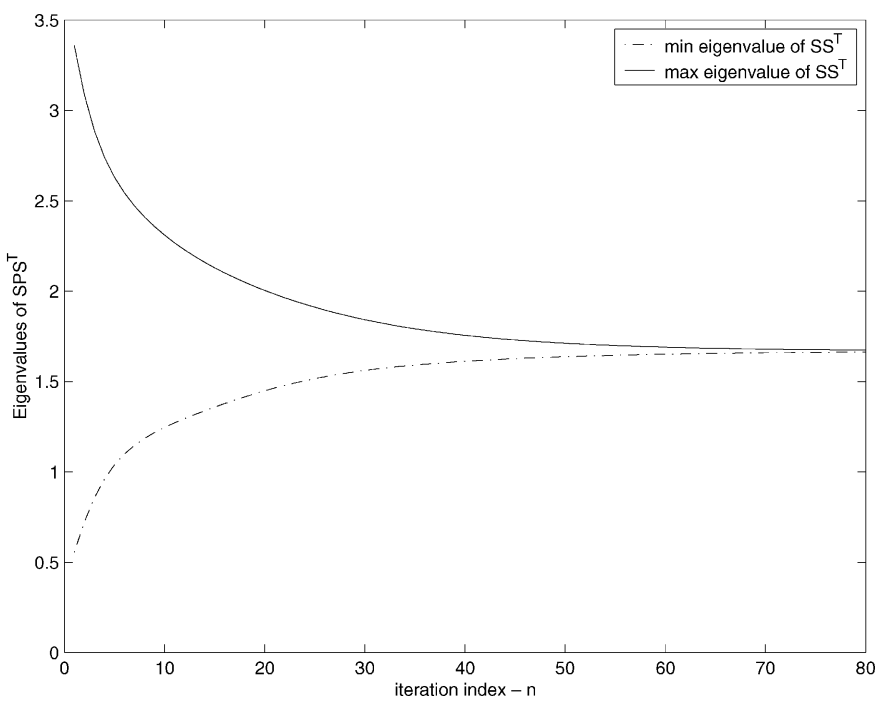

(b)

Fig. 1. (a) Total MSE and (b) minimum/maximum eigenvalues of $\mathbf{S S}^{\top}, N=6, K=10, p_{i}=1$.

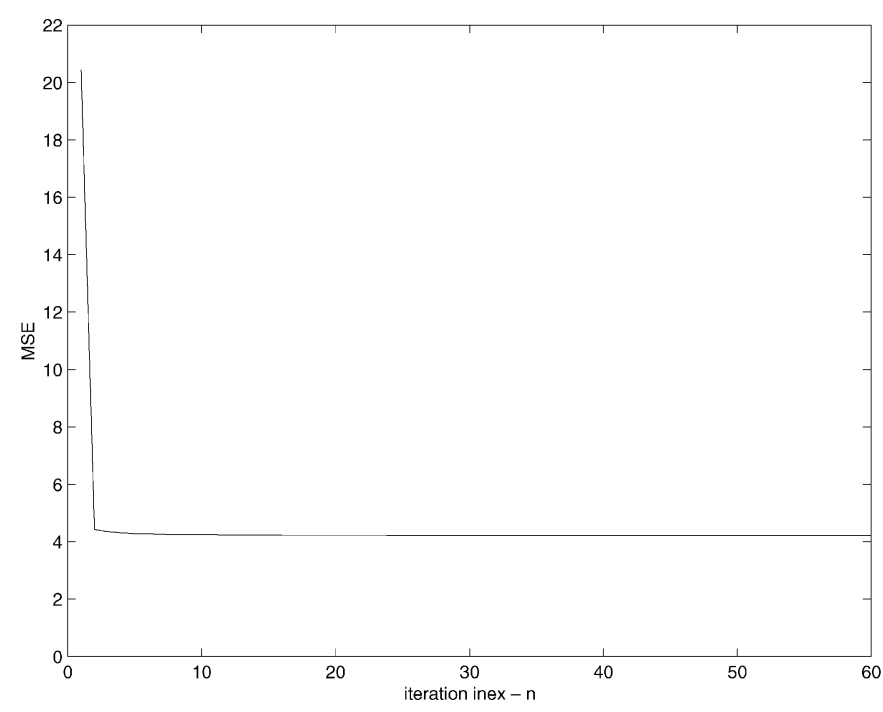

(a)

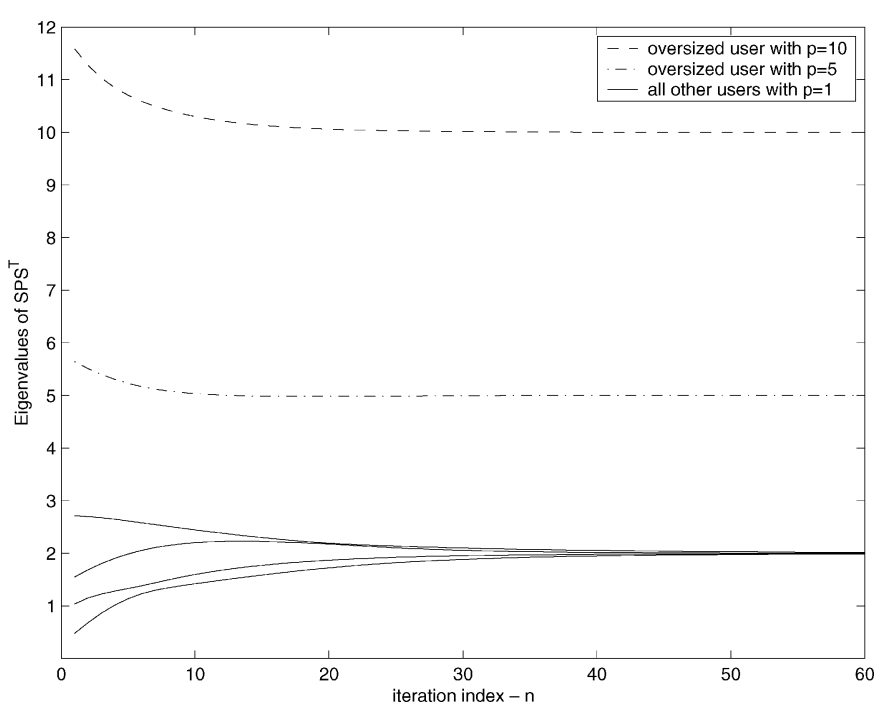

(b)

Fig. 2. (a) Total MSE and (b) eigenvalues of $\mathbf{S P S}^{\top}, N=6, K=10$, two oversized users.

the corresponding receivers in the $\epsilon$-neighborhood of the suboptimum fixed points of the algorithm which have strictly smaller MSE. This means that we can either escape the suboptimum fixed points of the algorithm using the escape methods of [19] and [20], or use a noisy version [19] of the proposed algorithm to ensure the almost sure convergence of the algorithm to globally optimum signature sequences and the corresponding receivers starting with arbitrary initial transmitters-receivers.

\section{NumeriCAL RESUltS}

In this section, we present numerical examples to support our analysis. We consider example CDMA systems with processing gain $N=6$ and investigate different scenarios. In all experiments, the initial signature sequences are created randomly, and all users update their receivers in parallel once followed by their update of the signature sequences in parallel once. Thus a total of $2 K$ updates are done between iterations $n$ and $(n+1)$.
Our first example is a system with $K=10$ users with equal received powers which we set to unity. Fig. 1 shows the total MSE which monotonically decreases and converges to its minimum possible value, and the minimum and maximum eigenvalues of $\mathbf{S S}^{\top}$ which converge to $K / N=1.67$, verifying that the resulting signature sequence set satisfies $\mathbf{S S}^{\top}=K / N \mathbf{I}_{N}$, i.e., it is a WBE set.

Next, we consider the same system where two of the users' received powers are increased to $p_{1}=10$ and $p_{2}=5$. The remaining eight users have unit received powers. It is easy to see that, in this system, the two users with higher powers are oversized [8]. Thus, the optimum signature sequence set dedicates each of the oversized users their own signal dimensions, and the rest of the users are assigned generalized WBE sequences in the remaining four dimensions. The eigenvalues of $\mathbf{S P S}^{\top}$ with the optimum signature sequence set are $\lambda_{1}=10, \lambda_{2}=5$, and $\lambda_{k}=2$ for $k=3, \ldots, 6$. Fig. 2 shows the MSE and the evolution of the eigenvalues of $\mathbf{S P S}^{\top}$ and their convergence to the 


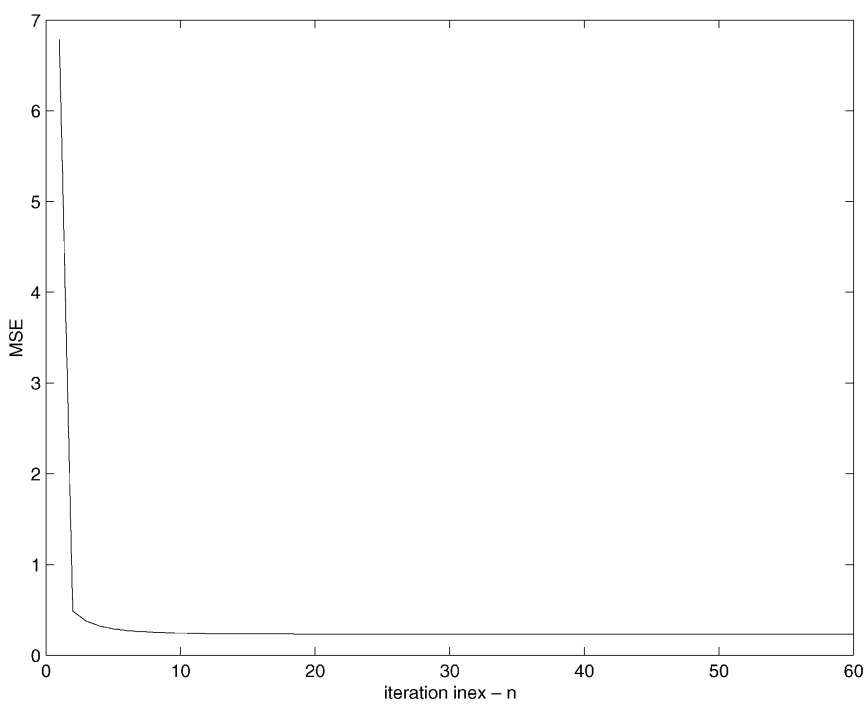

(a)

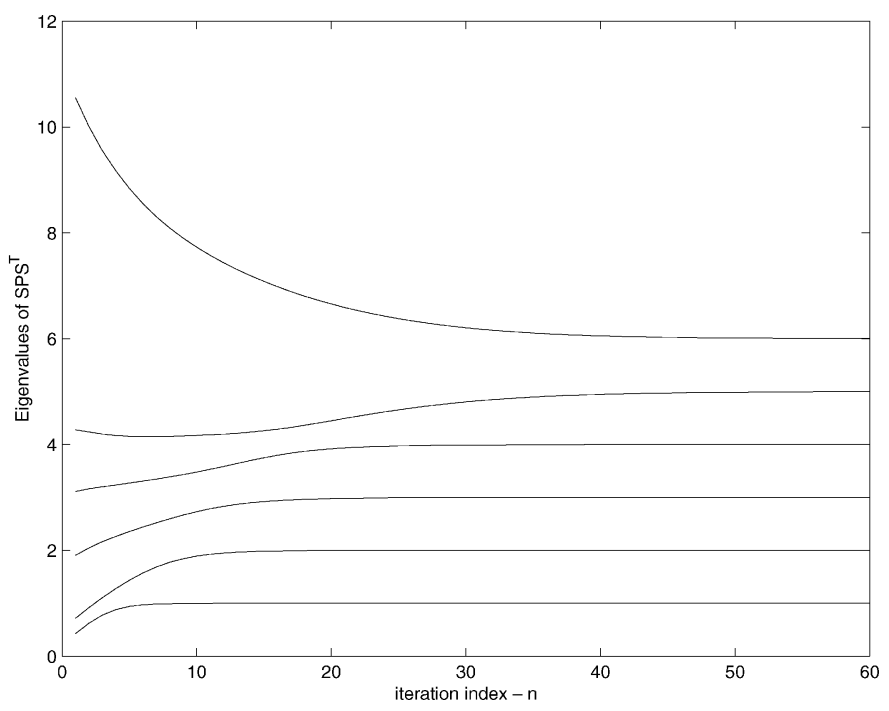

(b)

Fig. 3. (a) Total MSE and (b) the eigenvalues of $\mathbf{S P S}^{\top}, N=6, K=6, p_{i}=i$.

optimum values as we run the iterative transmitter receiver optimization algorithm.

In the last example, we have $K=6$ users and thus the system is not overloaded. In this case, the optimum signature sequences are orthogonal to each other irrespective of the received powers. For this example, we have used the received powers of $p_{i}=i$ for $i=1, \ldots, 6$. Fig. 3 shows the MSE values as we run the iterative algorithm converging to the sum of six single-user MSE values as the signature sequences converge to an orthonormal set, as well as the evolution of the eigenvalues of $\mathbf{S P S} \mathbf{S}^{\top}$ converging to the optimal values, i.e., the received powers of the users.

\section{CONCLUSION}

The algorithm proposed in this paper is one where users iteratively update their transmitters (signature sequences) and receivers. The receiver updates depend on the signature sequences of all users while the transmitter updates depend on the receivers of all users. Thus, users do not need to be scheduled for transmitter updates as is required for the convergence proof of the TWSC minimization based algorithms [9]-[11]. Furthermore, the algorithm can be implemented online and receivers can be constructed in an adaptive [6], [12], [13] or blind-adaptive [14] fashion at each iteration of the algorithm. The algorithm is shown to converge to the joint optimum transmitters and receivers with probability one with random initial points. We have presented numerical results that support the analysis under different system scenarios.

\section{REFERENCES}

[1] S. Ulukus and A. Yener, "Iterative joint optimization of CDMA signature sequences and receiver filters," in Proc. Conf. Information Sciences and Systems, Mar. 2002.

[2] 1 , "Iterative transmitter and receiver optimization for synchronous CDMA systems," in Proc. IEEE Int. Symp. Information Theory, June 2002.

[3] D. N. C. Tse and S. V. Hanly, "Linear multiuser receivers: Effective interference, effective bandwidth and user capacity," IEEE Trans. Inform. Theory, vol. 455, pp. 641-657, Mar. 1999.
[4] S. Verdú, "Capacity region of Gaussian CDMA channels: The symbolsynchronous case," in Proc. 24th Annu. Allerton Conf. Communication, Control and Computing, Oct. 1986, pp. 1025-1034.

[5] P. Viswanath, V. Anantharam, and D. Tse, "Optimal sequences, power control and capacity of spread-spectrum systems with multiuser receivers," IEEE Trans. Inform. Theory, vol. 45, pp. 1968-1983, Sept. 1999.

[6] U. Madhow and M. L. Honig, "MMSE interference suppression for direct-sequence spread-spectrum CDMA," IEEE Trans. Commun., vol. 42, pp. 3178-3188, Dec. 1994.

[7] M. Rupf and J. L. Massey, "Optimum sequence multisets for synchronous code-division multiple-access channels," IEEE Trans. Inform. Theory, vol. 40, pp. 1261-1266, July 1994.

[8] P. Viswanath and V. Anantharam, "Optimal sequences and sum capacity of synchronous CDMA systems," IEEE Trans. Inform. Theory, vol. 45, pp. 1984-1991, Sept. 1999.

[9] S. Ulukus, "Power control, multiuser detection and interference avoidance in CDMA Systems," Ph.D. dissertation, Dept. of Electrical and Computer Engineering, Rutgers-The State University, 1998.

[10] S. Ulukus and R. D. Yates, "Iterative construction of optimum signature sequence sets in synchronous CDMA systems," IEEE Trans. Inform. Theory, vol. 47, pp. 1989-1998, July 2001.

[11] C. Rose, S. Ulukus, and R. D. Yates, "Wireless systems and interference avoidance," IEEE Trans. Wireless Commun., vol. 1, pp. 415-428, July 2002.

[12] P. B. Rapajic and B. S. Vucetic, "Adaptive receiver structures for asynchronous CDMA systems," IEEE J. Select. Areas Commun., vol. 12, pp. 685-697, May 1994.

[13] S. L. Miller, "An adaptive direct-sequence code-division multiple-access receiver for multiuser interference rejection," IEEE Trans. Commun., vol. 43, pp. 1746-1755, Feb./Mar./Apr. 1995.

[14] M. Honig, U. Madhow, and S. Verdú, "Blind adaptive multiuser detection," IEEE Trans. Inform. Theory, vol. 41, pp. 944-960, July 1995.

[15] A. W. Marshall and I. Olkin, Inequalities: Theory of Majorization and its Applications. New York: Academic, 1979.

[16] D. Bertsekas, Nonlinear Programming. Belmont, MA: Athena Scientific, 1995.

[17] G. S. Rajappan and M. L. Honig, "Spreading sequence adaptation for DS-CDMA with multipath," IEEE J. Select. Areas Commun., vol. 20, pp. 384-395, Feb. 2002.

[18] W. M. Jang, B. R. Vojcic, and R. L. Pickholtz, "Joint transmitter-receiver optimization in synchronous multiuser communications over multipath channels," IEEE Trans. Commun., vol. 46, pp. 269-278, Feb. 1998.

[19] P. Anigstein and V. Anantharam, "Ensuring convergence of the MMSE iteration for interference avoidance to the global optimum," IEEE Trans. Inform. Theory, vol. 46, pp. 873-885, Sept. 2000.

[20] C. Rose, "CDMA codeword optimization: Interference avoidance and convergence via class warfare," IEEE Trans. Inform. Theory, vol. 47, pp. 2368-2382, Sept. 2001. 